\title{
Les milieux de vie de Stenasellus virei Dollfuss, 1897 (Crustacé Asellote troglobie): données préliminaires
}

\author{
par GUY MAGNIEZ ${ }^{11}$
}

Avec planches $125(1)-126(2)$

\section{Généralités:}

Les publications les plus récentes (Lanza, 1966), font état d'une vingtaine de stations seulement pour Stenasellus virei. Elles tiennent compte uniquement des citations de Stammer (1936), Racovitza (1950), Chappuis et Jeannel (1951), Angelier (1953), Husson (1957) et Braga (1962). Depuis la publication de la 9e Enumération des grottes visitées de Biospeologica (Coiffait, 1959), le nombre des stations s'est, en réalité, accru à un rythme rapide puisque, dans le courant de 1967, il nous a été possible d'en recenser 77 (Magniez, 1968). Ce progrès a été enregistré principalement grâce à l'activité des membres permanents ou temporaires du Laboratoire souterrain de Moulis, mais aussi à la suite des explorations de chercheurs isolés et de spéléologues étrangers et français.

Parmi ces stations, 56 sont des grottes, les autres correspondent à des prélèvements effectués dans les nappes phréatiques superficielles ou profondes (puits, filtrages de sources) et dans le sous-écoulement de rivières aquitaines (Tarn, Salat et ses affluents, etc. . .)

Les plus récentes prospections semblent montrer que la présence des Sténaselles dans les domaines interstitiels n'est pas un accident local. L'espèce paraît coloniser des milieux hypogés très variés dont nous allons essayer de dégager sommairement quelques caractères. Il sera possible de tirer de cette étude quelques conclusions préliminaires sur le statut de cet Asellote souterrain (systématique de détail en liaison avec les dissociations écologiques et géographiques dans l'aire de répartition, vie cavernicole et comportement fouisseur de l'espèce, variations possibles de l'aire au cours des temps et hypothèses sur les origines et le mode de colonisation des eaux douces hypogées par les Stenasellidae).

1) Laboratoire de Biologie Animale et Générale, Faculté des Sciences, 6, Bd. Gabriel, 21 Dijon, France. 


\section{Classification des stations; les biotopes de St. virei:}

L'énumération des stations ${ }^{1)}$ suggère immédiatement leur répartition en 3 ensembles distincts:
A. Les grottes,
B. Les nappes superficielles (le milieu hypotelminorhéique).
C. Le sous-écoulement des rivières (le milieu hyporhéique).

\section{A. Les grottes:}

Par un curieux hasard, il a fallu attendre 1949, l'espèce étant alors connue depuis plus de 50 ans et dans 19 cavités différentes, pour que $S t$. virei révèle sa présence dans un milieu tout différent (puits). C'est à la suite de l'observation des biotopes du Crustacé dans 6 grottes seulement, mais dans des conditions écologiques très comparables, que Fage et Racovitza purent le retrouver à Padirac en 1913, dans un biotope considéré alors comme «normal», c'est à dire le fond limoneux de mares d'eau stagnante, bien pourvues de débris ligneux. L'habitat correspondant à la découverte originelle (les eaux courantes du ruisseau de la Fontaine) ne devait être qu'accidentel.

Le nombre des grottes qui abritent St. virei ayant dépassé 55 dans le courant de 1967, il convient maintenant d'essayer d'établir une classification écologique de ces stations.

\section{Flaques argileuses isolées:}

Un certain nombre de cavités visitées se présentent comme des grottes sèches, ne possédant qu'un seul amas d'eau trés localisé, en général permanent, sauf exceptionnelle sécheresse de fin d'été. Dans ce cas, le limon du fond reste malgré tout très humide et plastique: grottes du Mont de Chac (3), de Lespugne (11), de Peyort (12), Haiouat de Pelou (13), de Liqué (32) et Tute de Jovis supérieure (27) par exemple. L'alimentation en eau, toujours modeste, est fournie par des suintements temporaires ou permanents, provenant des niveaux formant le toit de la grotte ou de condensations de la voûte. L'évacuation ne doit fonctionner que très temporairement à la saison humide. Le reste de l'année, le niveau de l'eau résulterait d'un équilibre entre un apport quantitativement limité et les fournitures à l'atmosphère de la cavité. Le bilan étant faiblement négatif, on assiste à une baisse lente du niveau de la flaque, du gour, du lac envisagés, jusqu'à la fin de l'été; bais-

\footnotetext{
1) Lorsque leur nom sera cité, il pourra être suivi d'un nombre entre parenthèses, correspondant au numéro d'ordre de la station dans la liste chronologique publiée par ailleurs. Voir: Magniez G. Les stations de Stenasellus virei Dollfus (Crustacé Isopode troglobie). Sous le Plancher, Dijon, (1967, VI, 3, 36-48).
} 
se suivie d'une remontée automnale et hivernale. Un cycle annuel relativement régulier semble donc exister.

Un tel système paraît, au premier abord, isolé des circuits normaux des eaux, surtout vers l'aval. Il peut être installé dans les grottes fossilisées du point de vue hydraulique. Cet aspect des choses est naturellement purement actuel, c'est à dire transitoire à l'échelle des temps géologiques. Une étendue d'eau de ce type peut abriter des Sténaselles, mais l'importance numérique et la densité des animaux peuvent varier dans de larges limites.

a) Flaques très petites: (de l'ordre de quelques dm2) pauvres en apports de nourriture. Quelques individus adultes sont capables d'y subsister, souvent dans des conditions précaires. Il suffit parfois d'y prélever tous les individus visibles pour faire disparaître purement et simplement la station. Dans ce cas, on est en droit de se demander si les Crustacés n'ont pas accès en de tels points seulement lorsque des conditions tout à fait favorables et rarement réalisées ont été réunies. Dans d'autres cas, on pourrait penser à un renouvellement pluriannuel, ou à un enrichissement annuel de la faune de la flaque. De toute façon, il est nécessaire d'imaginer qu'on se trouve en présence d'un abri qui recueille périodiquement quelques individus d'une population plus importante qui existe dans le milieu souterrain voisin de la grotte étudiée, mais inaccessible à l'investigation humaine.

b) Les flaques peuvent atteindre une surface de 1 ou plusieurs $m 2$ : C'est le cas de l'amas d'eau de la grotte du Mont de Chac (3). Les apports de matière organique pouvant tenir lieu de nourriture sont à la fois notables et bien échelonnés dans le temps. Un tel milieu permet à un nombre plus important de Sténaselles de prospérer. Dans l'exemple cité, il y a toujours plusieurs dizaines d'individus visibles sur le limon du fond. La structure de la population est également différente. Les femelles ovigères y font fréquemment leur apparition, c'est à dire que la reproduction se manifeste dans un groupement de cette importance, qui comprend également des mâles adultes de tailles échelonnées entre 5 et 7,5 mm, par exemple. On y trouve aussi un pourcentage variable de femelles immatures (n'ayant point encore acquis leur oostégites) et des femelles adultes en phase de repos sexuel. Cette population possède une remarquable permanence au cours des temps. C'est ainsi que celle du Mont de Chac observée depuis une cinquantaine d'années, montre une relative stabilité numérique au cours de cette période. Elle sera étudiée en détail ultérieurement.

c) L'étendue des eaux habitées par le Crustacé peut se mesurer par dizaines de $m 2$ : c'est le cas de la grotte de l'Estelas (6). Les diverses observations laissent à penser qu'une telle accumulation d'eau et l'importance des réserves 
limoneuses du fond, ainsi que les apports de nourriture, tant animale que végétale, pourraient y permettre la vie de quelques milliers de Stenasellus, avec cette fois encore des adultes à tous les stades, montrant que les conditions écologiques sont telles qu'elles rendent possible la reproduction régulière de l'espèce. Nous reviendrons également sur la structure d'une telle population.

\section{Réseaux de circulations souterraines:}

Certaines des grottes étudiées se présentent comme des regards sur une circulation d'eau souterraine. Il peut s'agir de circulation rapide, à débit important: ruisseau ou rivière courant sur fond de galets, sur la roche nue ou concrétionnée ou garnie de placages d'argile compacte (réseau actif). Il peut s'agir aussi de circulations au débit extrêmement modeste, mais également permanentes, qui correspondent à des écoulements plus lents, laminaires par places et jalonnés de petites retenues limoneuses.

a) Cours d'eau souterrains: Nous savons que St. virei avait été découvert à quelques exemplaires, dans le ruisseau de la Fontaine à Padirac, après de fastidieuses recherches étalées sur plusieurs années. Fage et Racovitza s'étaient aperçu que ces eaux courantes, sur fond de cailloutis, n'abritaient que de rares individus. L'espèce semblait préférer les nappes calmes à fond limoneux. Cette observation a pu être répétée de nombreuses fois dans les Pyrénées. La découverte des Sténaselles dans les cours d'eau souterrains est très aléatoire et ces Crustacés y sont très disséminés (ils sont de plus peu visibles dans un tel milieu). C'est le cas des cavités suivantes: grottes de l'Eglise (18), de Terreblanque (23), de Béguet (25); Tute de Jovis inférieure (69), gouffre Lacoste (31), gouffre de la Coume Ferra (66), du Bourdal (36), Goueil di Her (65), etc. .. . Leur observation y est donc malaisée et les captures en eaux rapides sont peu fréquentes, insuffisantes en général pour servir de point de départ à des élevages d'adultes de la station.

Dans quelques cas cependant, des ruisseaux souterrains abritent une population plus dense (grotte de Lespiougue, 64, par exemple). Il faut alors remarquer que les eaux y courent sur un abondant remplissage de sables et graviers limoneux qui ménagent, au voisinage même de l'eau circulante, de nombreuses laisses tranquilles où les Sténaselles s'installent de préférence. Dans un autre cas (Plagnol de la Plagne, 29), le cours du ruisseau est formé d'une succession de flaques calmes, bien que l'eau y soit très claire et s'y renouvelle rapidement. Ces flaques sont réunies par des rapides étagés. Les zones d'accumulation de l'eau conservent pour une large part le limon noirâtre, riche en matières organiques végétales, apporté par les crues. C'est là que les Stenasellus s'installent, en nombre relativement important. 
Les interstices de ces sables et graviers bordant les cours souterrains constituent par eux mêmes d'importants biotopes de l'espèce. Dans les cas particuliers cités plus haut, ils sont en continuité presque directe avec les milieux phréatique et hypotelminorhéique surplombants, ce qui pourrait expliquer la richesse de ces peuplements cavernicoles continuellement enrichis par les apports de faune originaire des portions amont du réseau. A la grotte du Goueil di Her (65), une association analogue pourrait exister. En août 1967, le grand siphon de la cavité s'était trouvé réduit à une flaque de quelques $\mathrm{dm} 2$ et de quelques $\mathrm{cm}$ de profondeur. Il a été possible de vérifier que cette accumulation d'eau libre abrite une abondante population de Sténaselles de grande taille (jusqu'à $12,1 \mathrm{~mm}$ ), dont la composition sera étudiée par ailleurs. Rouch (1967) a constaté que cette grande masse liquide est liée à de très importants dépôts de sables et graviers noyés. St. virei colonise également ce milieu (la taille moyenne des individus y est plus faible). Cette cavité nous offre donc un exemple précis de biotope cavernicole complexe, à la fois d'eau libre et interstitiel.

b) Zones de suintements permanents: Sur des pentes argileuses ou rocheuses, en des points bien définis de certaines grottes (aven de Sainte Catherine, 39; grotte de Peyort, 12 ou cueva la Clotilde, 62, par exemple), existent de faibles, mais très stables écoulements qui fonctionnent en toute saison. Ces écoulements, laminaires par places, sont séparés par de minuscules replats où se forment des flaques de quelques $\mathrm{cm} 3$ seulement chacune. Ce système rappelle l'aspect de certains ruisseaux décrits plus haut (Plagnol de la Plagne), mais à une échelle très réduite et avec un débit infime. Les Sténaselles affectionnent particulièrement ce genre de milieu. On les y trouve disséminés, individu par individu, dans chacune des petites flaques étagées ou ils peuvent rester totalement immobiles pendant de longues heures. Ils sont aussi capables de se déplacer d'une flaque à l'autre, comme cela est bien connu pour les Amphipodes du genre Niphargus, bien que l'épaisseur de la lame liquide intermédiaire soit inférieure à celle de leur corps.

\section{Regards sur des réseaux noyés de fentes:}

Dans quelques grottes, les accumulations d'eau semblent en communication avec des systèmes de conduits latéraux ou sous-jacents, complètement noyés. Leur niveau paraît résulter d'un équilibre hydrostatique avec le réseau, en fonction de son alimentation et des possibilités de l'exutoire de ses eaux. C'est ce qui pourrait exister en certains points des grottes du Bédat (10), de Sainte Catherine (33) ou de Lespugne de Saleich (11), par exemple. Seule, une très faible proportion de la masse liquide peut alors être prospectée et l'importance de la population de Stenasellus ne peut se déduire du nombre des individus observables. 


\section{B. Les nappes phréatiques de plateau ou de pente:}

Dans des régions telles que les plateaux jurassiques bourguignons ou les Causses, dont le sous-sol est formé par d'importantes assises calcaires mésozoïques restées subhorizontales, la fraction des eaux de précipitation destinée à s'infiltrer peut le faire en empruntant d'emblée des voies karstiques. Dans la région nord-pyrénéenne habitée par St. virei, au contraire, les variations des sédimentations paléozoïque, jurassique et crétacée, ainsi que les jeux ultérieurs de la tectonique amènent l'existence fréquente de contacts non horizontaux entre des terrains imperméables ou à perméabilité diffuse et des couches calcaires perméables en grand. Il en résulte souvent pour les eaux, une disposition en série, sur le même système hydrographique, de parcours de type phréatique normal, puis de cheminements karstiques. Lorsque des couches schisteuses ou gréseuses dominent des niveaux calcaires, par exemple, la collecte des eaux d'infiltration s'y produit à travers le manteau d'altération superficielle et l'écoulement peut avoir à emprunter en aval des voies karstiques. Il existe ainsi parfois des nappes phréatiques perchées locales qui alimentent des grottes situées en contre-bas. Les eaux peuvent apparaître passagèrement en surface, à la faveur d'un horizon plus imperméable.

$\mathrm{Au}$ moyen de trous atteignant ces eaux dans leur site hypogé (Mestrov, 1962), ou lors d'inventaires de la faune rejetée par les exutoires en question (Bouillon, 1964), la présence de St. virei dans ces milieux phréatiques particuliers a été montrée à plusieurs reprises. Ainsi, l'espèce colonise les nappes superficielles alimentant les sources des pentes schisteuses crétacées qui dominent à l'Est la grotte-laboratoire de Moulis (Mestrov, 1962). St. virei est aussi présent dans les bassins de captage de plusieurs sources qui naîssent dans de telles conditions géologiques (dévono-carbonifère du massif de l'Arize, crétacé terminal du Plantaurel, Bouillon, 1964).

\section{Les nappes des vallées alluviales; le sous-écoulement des rivières:}

La découverte de Stenasellus virei dans un puits de la ville de Toulouse (G. Taberly, 2 juin 1949) est à l'origine des données actuelles sur ce nouveau biotope du Crustacé. Son importance résulte de plusieurs caractéristiques:

1. La station nouvelle se trouvait très loin de toutes les grottes connues à l'époque pour abriter l'espèce. Les plus proches d'entre-elles se situent à quelques $70 \mathrm{~km}$ plus au Sud. Il en résulte une considérable extension de l'aire de répartition de $S t$. virei.

2. Cette station se trouve éloignée de tout massif karstique d'une distance pratiquement égale à la précédente, ce qui paraît exclure tout peuplement du biotope par contamination latérale. 
3. Le puits du quartier Saint-Cyprien, où l'animal fût pêché, est creusé dans les graviers quaternaires grossiers des alluvions récentes de la Garonne, à quelques centaines de $\mathrm{m}$ du fleuve, ce qui écarte toute possibilité d'apport immédiat par les eaux épigées.

4. Les eaux phréatiques parafluviales paraissent circuler très aisément à travers ces masses de graviers (essais de pompage du puits et vitesse importante de remontée du niveau). Les interstices de ces graviers permettent donc l'existence et les libres déplacements de populations de Crustacés dont les adultes (femelles) dépassent $9 \mathrm{~mm}$ dans cette station.

5. Confirmations du peuplement de ces milieux en Sténaselles: St. virei vient, très récemment, d'être capturé dans les nappes alluviales du Tarn, en ville d'Albi (71), (Bou, 1966, 1967). La nouvelle station se trouve donc au niveau d'un grand affluent de la rive Nord de la Garonne, ce qui montre déjà que les observations faites à Toulouse ne sont pas celles d'une anomalie écologique tout à fait isolée. Le Crustacé a été retrouvé aussi dans un puits de la vallée alluviale du Salat (70), affluent pyrénéen de premier ordre de la Garonne, à quelques km au Sud de Saint-Girons, dans l'aire classique de répartition de l'espèce, bien qu'en milieu interstitiel parafluvial.

Les prospections les plus récentes ont été réalisées surtout par Bou et Rouch $(1966,1967)$, avec l'appui et les conseils des Professeurs DelamareDeboutteville et Vandel. Elles utilisent l'implantation de tubes d'acier perforés dans le lit même des cours d'eau et le pompage immédiat du liquide du sous-écoulement au sein des alluvions. Les premiers résultats de cette méthode sont très prometteurs (Bou et Rouch, 1967). Ainsi, St. virei a été retrouvé dans le sous-écoulement du Tarn à plus de $20 \mathrm{~km}$ en amont d'Albi (Embialet, 73), en un point où la rivière s'enfonce déjà dans les terrains du socle hercynien du Massif Central. Le Crustacé est également mis en évidence dans la circulation sous-jacente du Nert (75), affluent de second ordre du fleuve, sur des formations cristallines et paléozoïques du massif de l'Arize (Bou et Rouch, Gourbault et Lescher-Moutoué, 1967). Il est retrouvé dans les mêmes conditions sous le lit du ruisseau de Lachein (74), affluent de troisième ordre de la Garonne, à l'extrême Ouest de l'Ariège et en pays calcaire cette fois, les cavités des versants voisins étant peuplées de Sténaselles (Rouch, 1967).

Au cours de recherches indépendantes des précédentes, St. virei a été capturé dans les nappes alluviales de deux fleuves côtiers méditerranéens: la Têt (Angelier, 1949; station $\mathrm{n}^{\circ} 21$ ) et le Tech (Coineau, 1964; stations $\left.\mathrm{n}^{\circ} 53,56,58\right)$. Nous disposons donc à l'heure actuelle d'une dizaine de stations sous-fluviales ou parafluviales de St. virei. On peut qualifier ces stations d'hyporhéiques, au sens défini par Orghidan (1959). Elles sont réparties en des points très éloignés les uns des autres du bassin hydrographique de la Garonne et des deux fleuves côtiers méditerranéens cités. 
Leur nombre est désormais suffisant pour montrer que le biotope hyporhéique est normal pour St. virei. Des transitions semblent exister entre ce milieu et les eaux libres des grottes, comme le montre la capture de l'Isopode (Rouch, 1967), dans le milieu interstitiel souterrain de la grotte de Béguet (25) et du Goueil di Her (65).

\section{Conséquences de ces données écologiques nouvelles:}

Jusqu'en 1949, Stenasellus virei avait pu être considéré comme un Crustacé aquatique de moeurs purement cavernicoles. En effet, toutes les stations connues à cette date avaient été découvertes au cours de prospections des grottes pyrénéennes et cantabres ( $\mathrm{n}^{\circ} 2$ à 19$)$. Ce n'est donc que très récemment que la présence de l'espèce a été constatée dans les milieux de type hypotelminorhéique (Mestrov, 1962, Bouillon, 1964) et dans le domaine interstitiel des vallées formant le réseau de la Garonne (Taberly, 1949; Bou, 1966, Rouch, 1967). Les premiers de ces milieux peuvent constituer les portions amont extrêmes du système hydrographique, tandis que les seconds peuvent se situer en aval des grottes classiques à Stenasellus, en orientant l'ensemble selon le sens général de circulation des eaux.

Des perspectives nouvelles sont ainsi mises en évidence dans la connaissance écologique, éthologique et systématique de l'Isopode:

A. Sur le statut cavernicole de l'espèce,

B. Sur l'aire de répartition, ses limites, ses discontinuités apparentes, ses continuités possibles et sa signification actuelle,

C. Sur la systématique de détail de l'espèce polytypique St. virei, qui semble s'ordonner en plusieurs groupes diversifiés, coïncidant avec des localisations géographiques et écologiques différentes,

D. Sur certaines particularités du comportement de l'animal,

E. Sur les données relatives à l'origine du genre et plus précisément de l'espèce virei ou sur les processus qui lui ont permis de coloniser les milieux où elle vit actuellement. Les bases du problème se trouvent désormais largement renouvelées, ce qui permet de choisir les aspects les plus satisfaisants parmi les hypothèses déjà formulées par Arcangeli, Birstein, Chappuis, Husson, Racovitza, Stammer, etc. . . .

\section{A. Statut de St. virei:}

Traditionnellement, l'espèce fut considérée comme strictement cavernicole puisque, iusqu'à une date récente, toutes les stations répondaient au critére du milieu de vie correspondant. Au cours de ses recherches sur les biotopes de l'Oligochète Pelodrilus leruthi, Mestrov (1962) découvrit à plusieurs reprises le Crustacé dans le milieu hypotelminorhéique qu'il avait précédem- 
ment décrit. Cet auteur en conclut que $S t$. virei est un élément caractéristique de ces biotopes et, qu'à son avis, le peuplement des grottes par l'Isopode résulterait d'une migration ou d'un entraînement à partir du milieu hypotelminorhéique d'amont. St. virei ne peut donc plus être considére comme un véritable cavernicole, bien que son installation dans le domaine souterrain soit probablement très ancienne. Vandel (1964) confirme les affirmations de Mestrov et admet la possibilité que les eaux libres des grottes ne constituent qu'un habitat secondaire des Sténaselles alors que le domaine des nappes de pentes serait leur principal milieu de vie. Angelier (1953) pouvait encore affirmer que les Sténaselles étaient des formes de grottes ou de puits, rares dans les sables submergés. Depuis lors, l'espèce a été découverte, grâce à de nouvelles méthodes de capture (Coineau, 1964; Bou et Rouch, 1967), en divers endroits, très éloignés les uns des autres, dans les nappes de la Garonne et de ses affluents, comme dans celles du Tech et de la Têt, parfois très loin de tout massif karstique (en ce qui concerne le Roussillon, St. virei, capturé à plusieurs reprises en milieu interstitiel par Angelier et Coineau, n'a jamais, à notre connaissance, été mis en évidence dans une grotte des Pyrénées-Orientales, où les cavités sont pourtant nombreuses et ont été bien étudieés). L'espèce se comporte donc, dans ces régions aquitaine et méditerranéenne, comme une forme caractéristique des zones profondes des nappes parafluviales et du sous-écoulement des cours d'eau. Elle est donc dans ce cas un phréatobie typique. Si l'on reprend le schéma d'ensemble du réseau hydrographique, avec en amont, les portions hypotelminorhéiques (et les nappes superficielles d'altitude) où les eaux sont collectées, avec le transit éventuel du flux liquide en grotte, à la faveur d'un substratum de nature convenable et enfin avec l'émergence des eaux dans les vallées alluviales (une partie du liquide circulant en dessous du lit et latéralement à lui, dans les sables et graviers déposés antérieurement par la rivière), on constate que Stenasellus virei semble capable de s'installer dans toutes les portions hypogées de ce réseau hydrographique. Cette installation reste sous la dépendance de certaines conditions tout à fait locales du milieu (la granulométrie du dépôt, en particulier).

Cette constatation doit pourtant, pour le moment, conserver un aspect purement statique. Elle ne doit laisser préjuger en rien des rapports actuels pouvant exister ou non, entre des populations karstiques et hyporhéiques voisines, comme celles qui peuvent vivre au niveau d'une même section transversale d'une vallée alluviale, par exemple, (fig. 2). L'étude morphologique des individus de ces populations que leur écologie sépare, est indispensable à la solution du problème (Magniez, 1968). Néammoins, on peut admettre que le cavernicole qui se montre ou phréatobie, ou hyporhéiobie, selon les cas, n'a peut être pas perdu tout dynamisme adaptatif vis à vis des milieux variés que lui offrent les eaux douces hypogées. 
B. Aire de répartition de l'espèce:

\section{Limites géographiques et contexte géologique:}

St. virei est installé sur un vaste territoire qui s'étend en direction méridienne sur plus de $500 \mathrm{~km}$ et en latitude sur quelques $300 \mathrm{~km}$. Le Crustacé est présent à la fois sur les versants Nord et Sud des Pyrénées, bien que les populations connues actuellement soient plus nombreuses et diversifiées au Nord de la chaîne. Cette aire recouvre à la fois des domaines hydrographiques atlantiques et méditerranéens.

La récente assimilation à l'espèce virei des formes découvertes dans 7 grottes de la chaîne cantabre, autour de Santander (Magniez, 1966), montre l'existence d'un noyau de peuplement occidental. Ce noyau reste pour le moment isolé de l'aire principale, en l'absence de toute donnée sur les habitats extrakarstiques dans les zones intermédiaires.

L'aire principale nord-pyrénéenne, cantonnée au niveau des massifs calcaires de la partie centrale de la chaîne, s'étend largement sur le bassin aquitain en direction du Nord. Elle mord sur le massif cristallin et paléozoïque du Centre de la France au N. E. et atteint vers l'Est les plaines côtières du Roussillon, à la suite des découvertes récentes en milieu phréatique. Les limites exactes de cette aire ne peuvent être précisées actuellement. Elles ne le seront que progressivement, par l'étude systématique de la faune des nappes alluviales et du sous-écoulement du réseau de la Garonne, de l'amont vers l'aval, d'une part, et, d'autre part, par l'extension de ces prospections aux bassins de l'Adour à l'Ouest et des rivières méditerranéennes à l'Est. On distingue donc sur les versants atlantiques:

a) La forme cantabre qui habite les 7 grottes situées dans les bassins de plusieurs courts fleuves côtiers (rios Ason, Pas, Besaya).

b) Les formes cavernicoles et interstitielles aquitaines qui sont installées sur le bassin hydrographique de la Garonne proprement dit, sur celui de la Dordogne (Padirac, 1) et qui débordent sur le bassin de l'Adour vers Bagnères de Bigorre (Batsère, 57; Bédat, 10; Castel-Mouly, 8). et sur les versants méditerranéens:

c) Les populations phréatiques des bassins de la Têt et du Tech, qui sont aisément reconnaissables des précédentes.

d) La colonie du Solencio de Morrano (5), dans la province de Huesca, appartenant au bassin de l'Ebre. Les individus en sont identiques à ceux de la forme hyporhéique aquitaine.

Du point de vue géologique, il ne semble pas que les Sténaselles soient inféodés à des niveaux déterminés. Le cas des habitats interstitiels est 
particulier lorsqu'il s'agit de formations alluviales jeunes, basses terrasses et alluvions récentes, mais il ne doit laisser en rien préjuger de l'ancienneté de ce type de peuplement. La nature pétrologique des éléments (graviers, galets) formant les alluvions colonisées par l'espèce est des plus diverses, selon les localités.

Quant aux grottes qui abritent le Crustacé, beaucoup se creusent dans les calcaires des divers étages crétacés qui forment des reliefs très importants au Sud de la vallée de la Garonne. Les stations cantabres correspondent aussi à des cavités des formations urgoniennes. Pourtant, les masses calcaires ou dolomitiques du lias ou du jurassique moyen sont également représentées, bien que très dissemblables dans les Pyrénées (grottes de Moulis, 4 et de Gourgue, 15) et en Quercy (gouffre de Padirac, 1). Plus rarement, les terrains primaires ou tertiaires sont mis à contribution. Ainsi, la galerie de Couflens de Betmajou (39) a été forée dans des calcaires-griottes rouges et verts du devonien terminal, tandis que le Solencio de Morrano (5) se trouve dans le calcaire lutétien d'Espagne. La partie terminale du chaînon du Plantaurel, isolée au N. W. de la Garonne, est constituée par une ossature de calcaires daniens qui s'ennoient progressivement sous le cône détritique miocène du Lannemezan. Cette structure particulière abrite aussi St. virei dans ses eaux phréatiques en plusieurs points déjà connus (Bouillon, 1964), mais non en grottes.

Les biotopes de Stenasellus virei sont donc installés dans des terrains de tous âges, du primaire de la chaîne axiale aux alluvions modernes. Il ne semble pas exister de relation entre l'âge géologique et la présence des Sténaselles, ou tout au moins, la seule relation qui paraît évidente résulte de la plus ou moins grande aptitude de chacune de ces formations géologiques à se creuser de cavités karstiques ou à recéler des nappes phréatiques.

2. Continuité et discontinuités de l'aire:

a) L'expression même de Racovitza, dans la monographie des Stenasellus (1950) indique bien que l'espèce virei était encore considérée comme strictement cavernicole. L'idée de l'isolement des diverses populations au niveau des grottes qui abritent chacune d'elles se retrouve également à plusieurs reprises dans le texte de l'auteur. Il y emploie fréquemment l'expression: «la colonie» de telle grotte et, à l'époque, l'espèce pouvait passer pour une réunion artificielle d'un certain nombre de populations, totalement isolées les unes des autres, chacune dans une cavité particulière et éloignée de quelques $\mathrm{km}$ ou dizaines de $\mathrm{km}$ de la station voisine, en schématisant les choses à l'extrême.

b) La découverte de la vie phréatique de l'espèce, donc d'une certaine indépendance vis à vis des milieux karstiques et l'exploitation par Mestrov (1962) de cette donnée intéressante, permettent déjà d'imaginer pour St. virei, une répartition moins discontinue que celle qu'offrirait le seul habitat 
cavernicole. Néammoins, toutes les nappes superficielles de pente sont loin de fournir une faune de ces Crustacés et les captures réalisées à ce jour indiquent leur étroite localisation aux eaux souterraines de certaines pentes, de certains vallons, donc à des zones très limitées. Il ne faut ainsi accepter qu'avec certaines restrictions l'affirmation de Mestrov (1962): "Stenasellus virei peuple le milieu hypotelminorhéique; et c'est à partir de ce biotope qu'elle a gagné les ruisseaux et les gours souterrains». La première proposition ne peut s'appliquer pour le moment qu'à certains sites tout à fait localisés et favorables; il n'est pas certain qu'elle ait une valeur générale. La seconde est une simple éventualité, car les ruisseaux et les gours souterrains ont pu aussi être peuplés par des faunes venant de l'aval.

c) C'est en effet avec la mise en évidence de St. virei dans le sous-écoulement qu'apparaît un nouvel aspect du problème de la continuité de l'aire du Crustacé. Le fleuve et ses divers affluents de tous ordres creusent leur lit au milieu de leurs alluvions quaternaires et récentes. Un tel milieu doit présenter une remarquable continuité longitudinale, les courtes interruptions dues aux seuils rocheux mises à part. Par contre, les divers auteurs qui se sont préoccupés de l'existence de la faune interstitielle sont d'accord pour admettre que les caractéristiques granulométriques de l'alluvion sont un facteur fondamental du problème du peuplement (Prenant, 1932).

Parmi les exemplaires de St. virei capturés jusqu'à présent dans les nappes parafluviales, nous avons trouvé à la fois de jeunes individus ( 2 à $3 \mathrm{~mm}$, âge approximatif de $1-2$ ans) et aussi, pour certains prélèvements, des adultes (mâles, femelles ovigères) de 6 à $9 \mathrm{~mm}$ (puits à Toulouse, nappe du Tarn à Albi et Embialet, etc...). Dans le premier cas (Toulouse, 20), il est possible de constater que la nappe de la Garonne imprègne des dépôts de graviers (taille de 1 à quelques $\mathrm{cm}$ ), non colmatés, où la circulation se révèle très active. Dans le second cas, les abondantes formations de galets du lit du Tarn comprennent des éléments de taille encore supérieure, avec des interstices libres de tout colmatage par les phases fines, à part une fine pellicule limoneuse. Le fait que certaines des captures soient réalisées par tubage et pompage immédiats dans le sous-écoulement de la rivière (Bou et Rouch, 1967), élimine la possibilité de la présence d'individus de taille exceptionnelle, qui auraient pu croître en eau libre, comme ce serait le cas pour les captures réalisées au fond d'un puits maçonné. La présence, en plusieurs points des nappes alluviales de la Garonne, de la Têt et du Tech, de St. virei est un fait indiscutable et non plus une simple possibilité logique. De véritables populations interstitielles existeraient en ces points, d'autant que les lots recueillis renferment aussi bien des individus immatures de faible taille que des adultes dont la dimension n'est que légèrement inférieure à celle des cavernicoles. L'existence générale de formations alluviales dont la granulométrie permet l'installation de l'espèce, est également une réalité 
constatée. Par contre, nous ne pouvons qu'émettre des hypothèses sur la structure de détail et la continuité de ces dépôts à larges interstices, donc sur la continuité à petite échelle des peuplements de Sténaselles. En ce qui concerne les fleuves côtiers des Pyrénées-Orientales, Angelier a publié (1962) des courbes relatives aux dépôts du lit du Tech, qui mettent en évidence une importante prédominance des éléments de taille voisine et supérieure à $2 \mathrm{~mm}$ ( 60 à $70 \%$ en poids sont constitués par les catégories de 2 à $12 \mathrm{~mm}$ et au dessus), alors que la proportion tombe à des valeurs très basses pour les tailles inférieures à $1 \mathrm{~mm}$.

d) Malgré cette relative rareté des données et les nombreuses lacunes qui subsistent, il est possible d'esquisser une explication, ou les diverses explications possibles de l'existence des peuplements isolés.

d1) Continuité de peuplement de massif à massif dans la région pyrénéenne?

Les massifs nord-pyrénéens à ossature calcaire sont subdivisés d'Est en Ouest, par les vallées de la Garonne et de ses principaux affluents, en un certain nombre de blocs relativement isolés les uns des autres. Les nappes alluviales que recèlent ces vallées, dans la mesure où elles se trouvent peuplées, au moins localement, par St. virei, cessent de devoir être considérées obligatoirement comme des barrières entre les massifs karstiques voisins. Elles peuvent désormais passer, au même titre, pour des zones de contact entre les populations des deux versants. Il se peut aussi qu'elles fonctionnent (ou aient fonctionné dans le passé), dans certains cas, comme des centres de dispersion de la faune et contribuent (ou aient contribué) à alimenter périodiquement le peuplement des deux blocs encadrants. Au Nord des Pyrénées centrales, on trouve d'Est en Ouest, la succession suivante: massif de l'Arize, vallée du Salat, massifs d'Arbas et de Sourroque, haute vallée de la Garonne, massif de Nistos, vallée de la Neste d'Aure . . A Au delà de cette rivière, de part et d'autre de Bagnères-de-Bigorre, existent les stations françaises les plus occidentales que nous connaissions (Batsère, 57; Bédat, 10; Castel-Mouly, 8). L'ensemble appartient déjà au bassin de l'Adour, mais aucun indice ne permet encore d'affirmer que $S t$. virei colonise également les milieux hyporhéiques dépendant de cette rivière. Les prospections ultérieures permettront de préciser ce point.

Une division analogue quoique moins marquée, se retrouve dans le sens Nord-Sud. Ainsi, les massifs d'Arbas et de Sourroque sont-ils séparés par la vallée méridienne du Lez, affluent de second ordre de la Garonne. Le schéma se répète entre chaînon du Plantaurel et massif de l'Arize, tous deux également colonisés par St. virei et entre lesquels il reste à préciser l'importance des peuplements intermédiaires. 
d2) Les peuplements alluviaux du Tarn:

Ils seraient totalement isolés de toutes les autres stations si un lien naturel n'existait avec elles et d'abord avec celle de Toulouse, qui constitue comme un relai. Ce lien logique est constitué par les nappes alluviales du fleuve et de son affluent qui se rejoignent à environ $60 \mathrm{~km}$ en aval de Toulouse. Pour le moment, on ne peut toutefois pas affirmer que cet important territoire est toujours colonisé par le Crustacé. Il est aussi à remarquer que jamais St. virei n'a été capturé dans les cavités des régions karstiques situées au Nord et au Sud d'Albi, malgré de minutieuses recherches (Bou, 1966). Il se retrouve, par contre, dans le sous-écoulement du Tarn, à $20 \mathrm{~km}$ à vol d'oiseau, en amont d'Albi (beaucoup plus en tenant compte des nombreux méandres); en des points où la vallée de la rivière s'encaisse dans le socle hercynien du Massif Central (Bou, 1967), La répartition de St. virei semble donc, dans cette zone, totalement indépendante de celle des grottes locales. L'espèce s'y comporte comme si elle ignorait les milieux karstiques existants, d'autant que, quittant les terrains paléozoïques, le cours supérieur du Tarn se creuse, plus à l'Est, dans les grands Causses calcaires, dont les innombrables grottes n'ont jamais, elles non plus, fourni de Sténaselles. L'espèce ne paraît dépendre ici que du domaine interstitiel lié au cours d'eau.

d3) Les Sténaselles de Padirac:

La station originelle de St. virei présente toujours cet extrême isolement par rapport à celles de l'aire principale pyrénéenne densément pourvue. $\mathrm{Ce}$ fait n'a pas manqué d'intriguer les différents auteurs qui ont étudié l'espèce, dans la mesure où elle pouvait être considérée comme uniquement cavernicole. En effet, $200 \mathrm{~km}$ séparent Padirac des plus proches grottes souspyrénéennes à Sténaselles, occupés en partie par les vastes étendues molassiques sans cavités de l'Aquitaine. Mais, le gouffre de Padirac s'ouvre dans les importantes masses calcaires et dolomitiques jurassiques qui forment le causse du Quercy. Les cavités naturelles de ce domaine karstique sont très nombreuses, les circulations souterraines abondantes et multiples, l'ensemble a été prospecté avec soin depuis fort longtemps et pourtant, jamais l'Asellote n'y a encore été trouvé ailleurs qu'à Padirac. L'explication classique de cette présence exceptionnelle est que cette faune de Padirac représente un cas unique de ce peuplement ancien, antéquaternaire, du causse du Quercy, détruit partout ailleurs dans le massif, par les rigueurs du climat périglaciaire. Une seconde hypothèse, tout aussi plausible que la précédente peut être formulée: Stenasellus virei pourrait exister à Padirac, comme un représentant de la faune du sous-écoulement de la Dordogne ayant migré de quelques kilomètres à l'intérieur du réseau karstique. La Dordogne coule en effet à peu de distance du gouffre et les eaux de celui-ci se dirigent vers la rivière par des cheminements souterrains encore incomplètement accessibles à l'Homme (de Lavaur, 1950). 


\section{Signification de l'aire actuelle de répartition:}

Il est unanimement reconnu que les glaciations quaternaires, plus spécialement les 2 dernières, ont eu un profond retentissement sur l'extension des faunes cavernicoles, surtout en Europe. Pour St. virei, relicte de faune thermophile, il en a certainement été de même et l'aire actuelle ne représente sans doute qu'un résidu d'une plus vaste répartition tertiaire.

Cependant, dans la mesure où les Sténaselles ne peuvent plus être considérés comme des cavernicoles parfaits, strictement inféodés au milieu aquatique des grottes et incapables de tout dynamisme colonisateur, il conviendrait peut-être de tenir compte des autres facteurs qui ont pu jouer un rôle positif ou négatif dans les variations de l'aire, ainsi que des nouveaux éléments du problème.

a) Les périodes froides et sèches du würmien ont certainement exercé une influence néfaste sur la répartition du Crustacé dans tous les domaines où il se montre capable de vivre:

- Les rigueurs du climat et le déficit des précipitations ont dû être responsables de la dégradation et du blocage du fonctionnement des milieux hypotelminorhéiques, nappes aquifères superficielles, à une certaine altitude sur les versants pyrénéens, restreignant ainsi ce premier biotope des Sténaselles.

- Ces restrictions ont évidemment atteint, selon l'opinion classique, le domaine des cavernes, par la suppression de leur alimentation et de leurs réserves en eau libre.

- Enfin, la diminution des circulations hydrographiques a dû entraîner des baisses considérables du niveau des nappes phréatiques de vallées, d'autant que la baisse du niveau de base devait occasionner une plus forte ablation des alluvions en place. Néanmoins, les rigueurs du climat devaient être atténuées par la basse altitude de ces milieux et les Sténaselles ont dû y conserver davantage de chances de survie (Ciry, 1959, 1962).

b) En schématisant beaucoup les évènements, les derniers épisodes froids et secs du Würm furent suivis d'un réchauffement rapide, avec une transgression marine (flandrienne) corrélative. On y a relevé une période au cours de laquelle la température moyenne a surpassé de 2 à $3^{\circ}$ les moyennes actuelles, mais aussi des phases de pluviosité certainement très supérieure à l'actuelle.

L'ensemble de ces conditions nouvelles (réalisées il y a environ 7000 ans) a amené un considérable développement du milieu forestier et très certainement en corrélation:

- Une reprise et une extension considérable du fonctionnement des milieux de type hypotelminorhéique, au sens le plus général, 
- Une réapparition et un grand développement des circulations et collectes d'eau par les grottes, avec un important concrétionnement,

- Une reprise de l'alluvionnement et une remontée des diverses nappes alluviales, en conformité avec la remontée du niveau de base.

Il n'est pas impossible que l'espèce ait profité de ces conditions plus favorables pour s'étendre à nouveau et que le domaine actuel de $S t$. virei soit aussi, pour une certaine part, un territoire de recolonisation postglaciaire.

c) En poursuivant le raisonnement, on peut envisager des évènements plus récents encore: une légère baisse des températures moyennes, une certaine diminution de la pluviosité, le déboisement, les drainages aux périodes historiques et enfin les ponctions importantes dans les nappes alluviales, qui ont baissé de plusieurs mètres en certains points, avec parfois des contaminations d'origine industrielle par des substances nocives. Tout un ensemble de causes, les unes naturelles, les autres d'origine humaine, ont pu se conjuguer aux temps historiques pour restreindre à nouveau l'aire de peuplement du Crustacé, bien que certains autres faits, comme les apports de bois en grotte ou la réalisation de retenues d'eau (sources captées, rivières barrées) lui aient peut-être apporté quelques nouvelles possibilités d'expansion locale.

En définitive, il demeure bien difficile de faire la part des divers phénomènes, les uns favorables, les autres nuisibles, qui ont interféré (et qui continuent de le faire, pour certains) pour imposer à l'aire de répartition de $S t$. vire $i$ les caractères qu'elle montre aujourd'hui. Néanmoins, il faut admettre que cette aire ne possède pas une géométrie achevée, définitive. Elle n'est que l'aspect actuel, essentiellement transitoire du domaine colonisé par l'espèce.

C. Les apports à la systématique détaillée de l'espèce:

Le nombre des stations de St. virei s'étant beaucoup accru, l'espèce se révèle présente dans des biotopes bien différents. L'étude comparée des individus des diverses provenances a fait apparaître une importante variabilité de certains caractères externes. En utilisant plusieurs de ces caractères stables dans une population déterminée, mais dont les fluctuations semblaient propres à des groupes de populations, en relation avec leur milieu d'origine et leur situation géographique au sein de l'aire, il a paru possible de fragmenter l'espèce en plusieurs entités bien distinctes. Celles-ci peuvent être considérées, dans l'état actuel de la connaissance que nous en avons, comme des sous-espèces (géographiques ou écologiques) de Stenasellus virei, l'espèce étant dite polytypique (le sens de ces divers termes est celui utilisé par Mayr, en 1963, dans l'exemple classique de Phymatoniscus tuberculatus Racovitza, exposé par Vandel en 1953). Le choix de la sous-espèce jouant le rôle de type s'appuie d'abord sur des caractères structuraux, mais il est apparu aussi que c'est dans le biotope hyporhéique que pourrait se rencontrer le peuplement le plus homogène morphologiquement et accessoirement, 
celui qui représente la biomasse la plus importante pour l'espèce. On doit naturellement lier cette homogénéité au fait que, comparé aux autres milieux hypogés, le sous-écoulement du réseau hydrographique possède peut-être la meilleure continuité écologique et géographique.

5 types ou sous-espèces sont donc à retenir pour le moment (Magniez, 1968) au sein de l'espèce Stenasellus virei Dollfus, 1897

1. St. virei virei: forme-type de Padirac et du domaine hyporhéique nord-aquitain, avec des représentants en Espagne (7 stations): Lot: 1 Gouffre de Padirac; Huesca: 5 Solencio de Morrano; Haute-Garonne: 20 Puits Pons à Toulouse; Ariège: 46 Source à Durban-sur-Arize; Tarn: 71 Puits tubé en ville d'Albi; 73 Sous-écoulement du Tarn à Embialet; 76 Sous-écoulement du Tarn à Albi ...

2. St. virei hussoni: forme des grottes et du milieu hypotelminorhéique des Pyrénées centrales, décrite en détail par Racovitza (1950), sur des échantillons du Mont de Chac (3) et de l'Estelas (6), (56 stations):

Ariège: 4 Grotte de Moulis; 6 Grotte de l'Estelas; 9 Grotte du Tuc d'Audoubert; 12 Grotte de Peyort; 14 Grotte de Sainte-Hélène; 19 Puits de la Mate; 24 Ruisseau souterrain d'Aulot; 27 Tute de Jovis supérieure; 28 Tute de Coumaratte; 29 Gouffre du Plagnol de la Plagne 30 Grotte Caujolle; 31 Gouffre de Lacoste; 32 Grotte de Liqué; 33 Grotte de Sainte-Catherine; 36 Gouffre du Bourdal; 38 Grotte Foulquier à Salège; 39 Aven de SainteCatherine; 40 Source de Salège; 42 Grotte des Trois-Frères; 43 Grotte d'Audinac; 44 Fontaine des Oiseaux; 45 Source et abreuvoir de Peyort; 48 Source captée d'Arbosec; 52 Aven du Tuc des Mandres; 54 Gouffre du Sauvajou; 60 Source de Millas; 61 Fontaine de la Bièle; 63 Ruisseau de Gamas; 64 Grotte de Lespiougue; 66 Gouffre de la Coume-Ferra; 67 Grotte de Montagagne; 68 Rivière souterraine d'Aliou; 69 Tute de Jovis inférieure; 70 Puits à Eycheil; 72 Cigalère des Trinquets; Haute-Garonne: 3 Grotte du Mont de Chac; 7 Gouffre du Poudac-Gran; 11 Grotte de Lespugne de Saleich; 15 Grotte de Gourgue; 22 Grotte de la Maouro; 23 Grotte de Terreblanque; 25 Grotte de Béguet; 26 Gouffre de Béguet; 47 Source Hountalayrou; 49 Source de Borde de Darré; 50 Source de Boulognesur-Gesse; 51 Ruisseau souterrain de Saint-Paul; 55 Grotte de Ruisec inférieur; 65 Résurgence du Goueil-di-Her; Hautes-Pyrénées: 2 Grotte de Gargas; 8 Grotte de Castel-Mouly; 10 Grotte du Bédat; 13 Grotte de l'Haiouat de Pelou; 18 Grotte de l'Eglise de Bas-Nistos; 37 Grotte de la Tute du Chef; 57 Grotte de Batsère.

3. St. virei buchneri Stammer, 1936 (Magniez, 1966), forme cavernicole de la province de Santander (Espagne) (7 stations):

16 Cuevas de Altamira (cueva con pinturas et cueva sin pinturas); 17 Cueva de la Estaćion de Santa-Isabel; 34 Cueva de Cullalvera; 35 Cueva del Molino; 59 Cueva la Castañera; 62 Cueva la Clotilde. 
4. St. virei angelieri: forme interstitielle du Roussillon (4 stations):

Pyrénées-Orientales: 21 Nappe alluviale de la Têt à Thuès-les-Bains; 53, 56, 58, Prélèvements dans les nappes alluviales du Tech à Brouilla.

5. St. virei boui: forme caractéristique du sous-écoulement du Salat et des torrents affluents. Elle semble isolée écologiquement de $S t$. virei hussoni qui peuple le milieu karstique des massifs encadrants, (4 stations):

Ariège: 41 Galerie artificielle de Couflens de Betmajou; 74 Nappe alluviale du ruisseau de Lachein; 75 Nappe alluviale du Nert; 77 Nappe alluviale du Salat à Salau ...

\section{Ecologie et comportement fouisseur de St. virei:}

Dans toutes les grottes où l'espèce peut être trouvée sur des fonds limoneux, quels que soient le calme et l'épaisseur de la couche liquide, des orifices de terriers se remarquent. Il est facile d'observer le Crustacé entrant et sortant par les orifices. Dès 1912, Jeannel et Racovitza, qui avaient noté la présence de nombreux trous sur le fond des flaques à Stenasellus, s'étaient demandé si ces Isopodes s'enfouissaient en creusant eux-mêmes la vase du fond ou si, le plus souvent, ils se dissimulaient tout simplement dans des trous des Oligochètes aquatiques qui se trouvaient fréquemment dans les mêmes biotopes limoneux. Ces Oligochètes furent étudiés par Hrabe en 1958 et décrits sous le nom de Pelodrilus leruthi. Racovitza est revenu sur cette question des terriers, en 1950, dans sa monographie de Stenasellus. Il est alors beaucoup plus catégorique dans son affirmation: «j’ai pu me convaincre que ces trous que Stenasellus utilise volontiers pour s'y réfugier étaient faits par les Oligochètes. Je ne crois pas qu'ils soient le résultat de l'activité de l'Isopode». Il notait de plus que les appendices des Stenasellus ne montrent aucune spécialisation pour fouir le limon. Husson (1957), fut le premier à rectifier cette affirmation et à montrer que $S t$. virei creuse bien des terriers dans le limon ou l'argile, tant en milieu naturel qu'au laboratoire. Cette aptitude à fouir les dépôts ou les roches plastiques est très développée. En quelques dizaines de minutes, un adulte est capable de se dissimuler dans la cavité qu'il vient de creuser dans l'argile cohérente garnissant le fond d'un récipient. Lorsqu'un groupe de Sténaselles est installé dans un tel récipient depuis quelques semaines toute la couche superficielle de l'argile se trouve ameublie par le travail incessant, par les passages répétés des Crustacés et par le transit d'une partie de la substance minérale par leur tube digestif. Meștrov (1962) reprend cette observation et la confronte avec les siennes sur l'habitat hypotelminorhéique de St. virei. Dans ce milieu, formé de limons et d'argile, riche en matière organique végétale, parcouru par le chevelu des racines, l'eau d'infiltration perfuse lentement par un réseau de canalicules. Stenasellus pourrait y exercer son activité fouisseuse avec 
bénéfice, afin de rechercher les emplacements qui lui sont le plus favorables. Le comportement fouisseur de l'espèce apparaît comme une nécessité, dès lors qu'elle fréquente les nappes aquifères de pentes et non plus seulement les eaux libres des grottes et les réseaux noyés de fissures des calcaires. Il est aussi une indication de la possibilité qu'a le Crustacé d'habiter les formations de galets et de graviers des vallées, formations noyées dans les nappes parafluviales. Ces graviers sont souvent plus ou moins colmatés par des phases fines au sein desquelles nous pouvons imaginer les Sténaselles, déblayant des galeries, avec la même activité qu'ils manifestent en présence d'argile de grottes. Leur remarquable aptitude au fouissement permet de penser que l'espèce pourrait posséder une capacité d'aménagement de son milieu de vie, au sein des alluvions fluviatiles, ainsi que des possibilités de migration souterraine, qui ont certainement été sous-estimées jusqu'alors.

\section{E. Les nouvelles données écologiques et l'origine des peuplements hypogés de}

St. virei; conclusion:

La question du processus de pénétration des Sténaselles dans les eaux douces hypogées et celle de l'âge de cette invasion, ont été débattues par de nombreux auteurs, qui ont pris, parfois, des positions catégoriques en faveur de telle hypothèse, ou de telle autre, radicalement opposée. Ici encore, il faut remarquer que les idées émises l'ont été en fonction de l'habitat strictement cavernicole attribué alors à l'animal. C'est dire que les raisonnements, appliqué d'ailleurs à plusieurs espèces du genre, (sp. virei, racovitzai, buili, asiaticus, etc ...) s'établissaient sur des bases systématiques, écologiques et biogéographiques ou très incomplètes, ou partiellement erronées.

Dollfus (1897) laisse à penser, sans l'affirmer, que nous nous trouvons en présence d'une forme-relique de Crustacé, amené par quelque trans gression des mers tertiaries vers les Causses. Hansen (1904) montre qu'il s'agit d'un type extrêmement archaïque d'Asellote, présentant quelques affinités avec des formes littorales de mers tropicales, mais il ne se prononce pas sur la question du passage d'un milieu à l'autre d'une éventuelle lignée, étant uniquement préoccupé par les problèmes de systématique du groupe. Stammer (1936) voit en Stenasellus un relicte d'une faune d'eau douce tertiaire, dont des espèces auraient subsisté en Europe méditerranéenne, en migrant dans les eaux souterraines à la suite de l'établissement de conditions climatiques défavorables. Cette hypothèse est largement développée par Chappuis (in Racovitza, 1950), qui la complète au moyen d'une hasardeuse supposition faisant intervenir des migrations de faune, afin d'expliquer les peuplements insulaires et de relier des portions continentales aujourd'hui 
séparées par des étendues marines. Certains points de l'hypothèse sont appliqués par Birstein (1951) à l'origine du St. asiaticus de Turkménie ou repris par Husson (1957).

Arcangeli (1938), par contre, considérant que Stenasellus, relicte cavernicole typique, possède des caractères morphologiques qui lui valent de représenter une famille d'Asellotes distincte des Asellidae, imagine que les Sténaselles cavernicoles peuvent parfaitement dériver de formes marines préadaptées et euryhalines, qui auraient colonisé le domaine karstique continental en empruntant le réseau de fissures qui fait communiquer les eaux marines littorales et le milieu aquatique hypogé. Il convient de reprendre point par point ces affirmations contradictoires et plus particulièrement leur application à l'espèce virei. En effet, la question devra être étudiée ultérieurement au niveau du genre et du groupe des Stenasellidae, en tenant compte de tous les arguments écologiques, biogéographiques, morphologiques et biologiques qui auront pu être rassemblés.

1. Le principe de la séparation de Stenasellus virei et des formes parentes, des Asellides vrais (Asellus, Proasellus, Baicalasellus, Synasellus, etc....), n'a fait que s'affirmer (Dudich, 1924; Arcangeli, 1938; Vandel, 1964; Magniez 1966; Lanza, 1966), basé sur des arguments morphologiques importants (structure de l'antenne, des pléonites I et II, de leurs appendices, nombre de caecums entériques, etc. . .), ce qui a mené à la reconnaissance de 5 genres de Stenasellidae (Johannella, Metastenasellus, Parastenasellus et Magniezia africains, plus Stenasellus périmésogéen). Peu à peu, l'idée se dégage donc que le groupe comporte des formes originales, dont l'espèce de Padirac est un exemple typique, qui ont colonisé les habitats dulçaquicoles hypogés, indépendamment des Asellides vrais (ce groupe étant certainement luimême hautement polyphylétique). Cette indépendance se conçoit à la fois dans l'espace et dans le temps.

2. Le fait que Stenasellus représente, en de nombreux points, une forme relicte est peu douteux, mais il n'existe aucune preuve qu'il s'agisse des restes d'une faune tertiaire épigée, qui aurait été détruite par les glaciations quaternaires. Rien n'indique que $S t$. virei ait subi un passage du milieu épigé d'eau douce au milieu hypogé sous-jacent, aussi récent, avec les conséquences anatomiques qui en découlent. Par contre, un tel processus est certainement celui qui a atteint les Proasellus apparentés à l'espèce coxalis. Il pourrait comporter les phases suivantes, à partir de l'établissement de la forme-souche épigée, sur un vaste territoire périméditerranéen, en eaux douces et en période de climat favorable à sa pullulation (tertiaire?):

a) Apparition de variants (dépigmentés, anophtalmes) parmi ces populations florissantes, variants suceptibles de vivre en eaux hypogées sans dommage. 
b) Dissociation écologique (plus ou moins rapide et complète) des 2 formes et installation des dérivés en milieu hypogé, avec migrations horizontales possibles vers les points les plus favorables de ce milieu hypogé.

c) Disparition locale ou généralisée de la forme oculée de surface, par suite des conditions climatiques devenues défavorables (glaciations, asséchement périodique, etc. ..).

d) Maintien local jusqu'à nos jours des formes dérivées dans certains milieux souterrains favorables.

Dans le cas de Stenasellus, un tel schéma ne paraît pas convenable. Il semble plutôt que la vie cavernicole et surtout interstitielle, représente un état extrémement ancien, peut-être un état d'origine. Stenasellus virei pourrait bien s'être installé en eaux douces en tant que phréatobie et n'être jamais dérivé de souche dulçaquicole épigée. D’ailleurs, même dans le cas des formes africaines, qui n'ont jamais eu à souffrir de glaciations, il n'existe aucune forme épigée connue, voisine systématiquement, dans laquelle on puisse trouver la souche des types anophtalmes. Les variations climatiques quaternaires peuvent donc tout au plus être responsables de restrictions (vraisemblablement considérables), de l'aire de répartition de St. virei (et de celle des autres espèces eurasiatiques), mais non de la disparition généralisée d'une hypothétique souche épigée, que l'on devrait détecter en tous points où les rigueurs du climat étaient très atténuées (Côte d'Ivoire, Congo) de même que l'on retrouve l'épigé Proasellus coxalis en Afrique du Nord et en Europe méditerranéenne et insulaire.

3. La répartition connue des diverses espèces de Sténaselles, avec des représentants insulaires et continentaux, est un bon argument en faveur de l'antiquité de leur installation en milieu hypogé. Mais ce sont aussi des relictes thermophiles (Vandel, 1964). Plusieurs formes vivent encore dans des eaux dépassant $20^{\circ}$ (la dernière décrite, St. pardii Lanza, 1966, est installée dans les eaux hypogées de Somalie, région subéquatoriale au climat extrêmement chaud; leur température atteint $29^{\circ}$, d'après Stefanini, 1916) ou dans des sources thermales des pays eurasiatiques dont la température moyenne annuelle est très inférieure à $20^{\circ}$. Il n'est absolument plus nécessaire de lier leur installation en eau douce à l'âge du karst pyrénéen, par exemple, puisque l'habitat cavernicole a de fortes chances d'être secondaire par rapport à celui du sous-écoulement. Ce dernier s'est bien entendu constamment modifié au cours des temps géologiques tertiaires puis quaternaires. Il a suivi les évolutions du réseau hydrographique, sous la dépendance du niveau de base général et des activités orogéniques, de la pédogenèse, de l'érosion et de l'alluvionnement. Ainsi, le fait que de nombreuses populations actuelles soient installées dans les alluvions récentes des rivières modernes n'est, en aucune façon, un obstacle à l'ancien- 
neté de l'animal dans un tel milieu, mais sans doute un aspect du dynamisme colonisateur de l'espèce. L'âge de cet établissement, en un lieu donné, pourrait donc être indépendant, aussi bien de celui de la formation géologique qui l'abrite, que de celui du karst qui a pu s'y établir.

4. Le passage de l'éventuelle souche de Stenasellus virei, des eaux marines aux eaux libres des grottes n'a pas nécessité l'existence de communications à grande section entre le milieu littoral et les réseaux noyés de fissures des massifs calcaires, comme ce pourrait être le cas pour les Cirolanides ou les Sphéromiens cavernicoles (Racovitza, 1907), car il est prouvé que St. virei fréquente aussi couramment le domaine hyporhéique profond. Ainsi, le passage de cette lignée, du milieu marin aux milieux aquatiques des grottes et aux nappes phréatiques d'altitude, a pu se faire par des voies continuellement interstitielles: galets et graviers littoraux, formations deltaïques, par exemple, puis sous-écoulement des rivières et milieu hyporhéique des plus petits affluents, pour gagner les eaux des grottes et même, dans certains cas, les nappes superficielles alimentant les sources des pentes montagneuses.

Plus qu'à l'histoire des Aselles hypogés, celle de l'installation de St. virei dans ses milieux de vie, devra peut-être se comparer à celle des Microparasellidés ou des Microcerbéridés, donc de phréatobies d'origine marine typique.

Dijon, juin 1968

\section{RÉSUMÉ}

Stenasellus virei est désormais connu dans 77 stations (grottes, nappes phréatiques et sous-écoulement des rivières) du bassin aquitain, des Pyrénées centrales et orientales et d'Espagne. Un essai de classification des différents biotopes a été tenté et leurs caractéristiques sommairement exposées. Cette espèce cavernicole se présente actuellement sous un jour nouveau, tant écologique que systématique ou biogéographique.

\section{SUMMARY}

Stenasellus virei is now known from 77 localities (caves, phreatic waters and underflow of some rivers) of the eastern Aquitanian basin, central and eastern Pyrenees, and of Spain. A classification of the different biotopes of the species is attempted herein, and some of their characteristics are summarily described. This cavernicolous species can now be wiewed in a new light, as much ecological as systematic or biogeographic.

\section{BIBLIOGRAPHIE}

Angelier, E. (1953) - Recherches écologiques et biogéographiques sur la faune des sables submergés. Arch. Zool. exp. gen. Paris, 9o, 37-162.

Arcangel, A. (1938) - Stenasellus racovitzai Razzauti, Crostaceo Isopodo acquatico cavernicolo italiano. La famiglia Stenasellidae. Boll. Mus. Zool. Anat. comp. Torino, 46, $17-50$.

Birstein, J. A. (1951) - Faune de l'U.R.S.S., VIII5, Asellota, Moscou, 1-142.

BonNeT, A. (1953) - Les Crustacés aquatiques cavernicoles et la paléogéographie de l'Oligocène. Actes ler Cong. Intern. Spéléol. Paris, III, 55-60. 
Bou, Cl. (1966) - Faune souterraine du Sud-Ouest du Massif Central, I. Ann. Spéléol. Paris, 21/3, 689-706.

- (1968) - Faune souterraine du Sud-Ouest du Massif Central, II. Ann. Spéléol. Paris, (en préparation).

Bou, Cl. \& Boulllon, M. (1965) - Sur quelques cavités du massif de Sourroque (St-Girons, Ariège). Ann. Spéléol. Paris, 20/1, 55-63.

Bou, CL. \& Rouch, R. (1967) - Un nouveau champ de recherches sur la faune aquatique souterraine. C. R. Acad. Sc. Paris, 265, D, 369-370.

Boulllon, M. (1964) - Contribution à l'étude écologique des Amphipodes du genre Niphargus dans les Pyrénées centrales. Ann. Spéléol. Paris, 19/3, 537 bis $551 ; 19 / 4,813-818$.

Braga, J. M. (1962) - Sur la distribution géographique des Stenasellus de la péninsule ibérique et description d'une nouvelle espèce du genre. Ann. Fac. Sc. Porto, 84, 1-28.

Brehm, V. (1948) - Reflexiones sobre relaciones zoogeographicas de la fauna de agua dulce de la Peninsula Iberica. Inst. Biol. Apl. Barcelona, IV, 53-74, 1947.

- (1955) - Süßwasserfauna und Tiergeographie. Österr. Zool. Zeitschr. Wien, 6, $250-269$.

Caliman, T. (1967) - Recherches sur les Crustacés souterrains des sources du massif de Sourroque (Ariège). D. E. S. Zool. Fac. Sc. Univ. Toulouse, 1-70.

Chappuis, P. A. (1950) - La récolte de la faune souterraine. Notes Biospèol. Paris, $5,1-29$.

Chodorowski, A. (1961) - Recherches sur la faune aquatique des grottes dans les Pyrénées centrales. Actes IIIe Cong. Intern. Spéléol. Wien, A, p. 48.

- (1962) - Différences écologiques entre les populations d'Asellides cavernicoles. C. R. Acad. Sc. Paris, 255, 3487-3489.

CIRY, R. (1959) - Une catégorie spéciale de cavités souterraines: les grottes cutanées. Ann. Spéléol. Paris, 14, 1/2, 23-30.

- (1962) - Le rôle du froid dans la spéléogenèse. Spelunca, Paris, Mem. n 2, 29 - 34

Codreanu, R. (1961) - Sur le peulement en Triclades et Asellides d'eau douce de quelques îles méditerranéennes. In Coll. Intern. C. N. R. S. Banyuls-sur-Mer, $21 / 27$. sept. $1959, \mathrm{n}^{\circ} 94,163-179$.

Coiffait, H. (1959) - Enumération des grottes visitées, $1950-1957$ ( $9^{\circ}$ série). Arch. Zool. exp. gen. Paris, 97, $209-465$.

Coineau, N. (1963) - Présence du sous-ordre des Ingolfiellidae Reibisch (Crustacea Amphipoda) dans les eaux souterraines continentales de France. C. R. Acad. Sc. Paris, 257, 4229-4231.

- (1964) - A propos de quelques Crustacés interstitiels nouveaux des eaux littorales de Corse. Vie et Milieu, Paris, 15/3, 809-811.

Delamare-Deboutteville, Cl. (1957) - Lignées marines ayant pénétré dans les eaux souterraines continentales. Un problème de biogéographie actuelle. $C$. $R$. som. Soc. Biogèogr. Paris, 53-57.

- (1960) - Biologie des eaux souterraines. littorales et continentales. Suppl. Vie et Milieu, Paris, 9, 1-740.

Derouet, L. (1953) - Vie aérienne de quelques Crustacés aquatiques cavernicoles et épigés. Actes Ier Cong. Intern. Spéléol. Paris, III/3, 233-236.

Geze, B. (1947) - L'origine des eaux souterraines. Ann. Spèlèol. Paris, 2/I, 3-10.

Ginet, R. (1960) - Ecologie, éthologie et biologie de Niphargus (Amphipodes Gammaridés hypogés). Ann. Spéléol. Paris, 15, 127-376.

Gourbault, N. \& Lescher-Moutoue, F. (1967) - Sur la faune hypogée peuplant le sous-écoulement d'une rivière de moyenne altitude. C. R. Acad. Sc. Paris, $256, \mathrm{D}, 1813-1816$.

HANSEN, H. J. (1904) - On the morphology and classification of the Asellotagroup of Crustaceans, with descriptions of the genus Stenetrium Hasw. and its species. Proc. Zool. Soc. London, 2, 302-331.

Henry, J. P. \& Magniez, G. (1968) - Un nouvel Aselle hypogé d'Espagne: Proasellus cantabricus n. sp. Ann. Spéléol. Paris (sous presse). 
Husmann, S. (1967) - Die ökologische Stellung der Höhlen- und Spaltengewässer innerhalb des subterranaquatilen Lebensreiche. Intern. Journ. Spéléol. Lehre, 2/2 409-436.

Husson, R. (1957) - A propos de la biologie du Crustacé troglobie aquatique Stenasellus virei Dollfus. Ann. Univ. Saraviensis, Sc., IV, 74, 8259-8269.

- (1959) - Les Crustacés Péracarides des eaux souterraines. Considérations sur la biologie de ces cavernicoles. Bull. Soc. Zool. France, 84, 4, 219-231.

JeAnnel, R. (1943) - Les fossiles vivants des cavernes. Gallimard, Paris, 1-381.

- (1965) - La genèse du peuplement des milieux souterrains. Rev. écol. biol. sol, Paris, 2, 1, 1-22.

LANZA, B. (1966) - Stenasellus pardii sp. n. della Somalia e note sistematische su gli Stenasellinae (Crustacea Isopoda). Monit. Zool. Ital. Firenze, 74, 221 - 256.

Lavaur, G. de (1950) - Le gouffre et la rivière souterraine de Padirac. Ann. Spéléol. Paris, 5, 2/3, 51-84.

Leruth, R. (1938) - La faune de la nappe phréatique du gravier de la Meuse à Hermalle-sous-Argenteau. Bull. Mus. Hist. Nat. Belgique, 14, 41, 1-37.

- (1939) - Une méthode interéssante pour l'étude de la faune phréatique. Bull. Soc. Roy. Liège, 8, 136-141.

Magniez, G. (1966a) - Contribution à la systématique des Stenasellinae (Crustacea Isopoda) d'Afrique. Intern. Journ. Speleol. Lehre, 2, 1/2, 173-190.

- (1966b) - Les Sténaselles (Crustacés Isopodes troglobies) de la Province de Santander (Espagne). Ann. Spéléol. Paris, 21/4, 827-837.

- (1967) - Contribution des spéléologues à la connaissance de certains Crustacés des grottes cantabres. Sous le Plancher, Dijon, 5, 3/4, 30-41, 1966.

- (1968 a) - Les Stations de Stenasellus virei Dollfus (Crustacé Isopode troglobie). Sous le Plancher, Dijon, VI, 3, 36-48.

- (1968b) - L'espèce polyrypique Stenasellus virei Dollfus, 1897, (Crustacé Isopode hypogé). Ann. Spéléol. Paris, 23/2, 363-407.

MargalefF, R. (1953) - Los Crustaceos de las aguas continentales ibericas. Inst. Forest. Invest. exp. Minist. Agr. Madrid, 10, 1-243.

MARTel, E. A. (1925) - Le gouffre et la rivière souterraine de Padirac (Lot.) Delagrave, Paris, $1-176$.

Mayr, E. (1963) - Animals species and evolution. Harvard Univ. Press, USA,

Meštrov, M. (1962a) - Un nouveau milieu aquatique souterrain: le biotope hypotelminorhéique. C. R. Acad. Sc. Paris, 254, 2677-2679.

- (1962b) - Milieu d'origine de quelques espèces aquatiques souterraines. Spelunca, Paris, 4 e ser., Mem. $\mathrm{n}^{\circ} 2,84-88$.

- (1964) - Différences et relations faunistiques et écologiques entre les milieux souterrains aquatiques. Spelunca, Paris, 4e ser., Mem. n ${ }^{\circ}$ 4, 185-187.

Orghidan, T. (1959) - Ein neuer Lebensraum des unterirdischen Wassers: der hyporeische Biotop. Arch. f. Hydrobiol. Stuttgart, 55, 3, 392-414

Racovitza, E. G. (1907) - Essai sur les problèmes biospéologiques. Arch. Zool. exp. gen. Paris, 4, 6, 371-488.

- (1950) - Asellides - première série: Stenasellus. Arch. Zool. exp. gen. Paris, $87,1-94$.

Rouch, R. (1968) - Th. Zool. Ann. Spéléol. Paris, 23/I, (sous presse).

Stammer, H. J. (1936) - Eine neue Höhlenwasserassel aus Spanien, Stenasellus buchneri und die Verbreitung der Gattung Stenasellus. Zool. Anz. Leipzig, 114, $137-141$.

Trieu, R. (1943) - Contribution à l'étude géologique, biologique et bactériologique du gouffre de Padirac (Lot.) Couestant, Cahors, 1-134.

Trombe, F. (1952) - Traité de spéléologie. Payot, Paris, 1-376.

VANDEL, A. (1964) - Biospéologie - la biologie des animaux cavernicoles. Gauthier-Villars, Paris, $1-169$.

- (1965) - Sur l'existence d'Oniscoïdes très primitifs menant une vie aquatique et sur le polyphylétisme des Isopodes terrestres. Ann. Spéléol. Paris, 20/4, $489-518$. 
VirÉ, A. (1899) - Essai sur la faune obscuricole de France. Etude particulière de quelques formes zoologiques. Th. Fac. Sc. Univ. Paris, 1-157.

WoLFF, B. (1934/1938) - Animalium Cavernarum Catalogus. W. Junk, Berlin.

\section{EXPLICATIONS DES PLANCHES 125 (1) - 126 (2)}

Fig. 1: Carte schématique du Sud-Ouest de la France et du Nord de l'Espagne. Les cercles noirs représentent de stations interstitielles de Stenasellus virei, les cercles blancs les stations cavernicoles. La densité des stations connues étant très forte dans les massifs calcaires des Pyrénées centrales, entre Garonne et Salat, le nombre des cercles y est inférieur au nombre réel de stations. Les 5 sous-espèces de St. virei n'ont pas été distinguées les unes des autres. Les 2 stations de St. breuili et la station de St. buili ont été indiquées. $\mathrm{A}=$ Adour, $\mathrm{Ar}=$ Ariège, $\mathrm{D}=$ Dordogne, $\mathrm{E}=$ Ebre, $\mathrm{G}=$ Garonne, $\mathrm{L}=$ Lot, $\mathrm{S}=$ Salat, $\mathrm{T}=$ Tarn; $\mathrm{MC}=$ Massif Central; $\mathrm{Al}=\mathrm{Albi}, \mathrm{Bi}=$ Bilbao, $\mathrm{Bo}=$ Bordeaux, $\mathrm{P}=$ Padirac, $\mathrm{Sa}=$ Santander, To $=$ Toulouse, $\mathrm{Za}=$ Sarragosse. Les points d'interrogation signalent les régions où les prospections ultérieures pourraient éventuellement révéler la présence des Sténaselles.

Fig. 2: Schéma théorique situant côte à côte les différents biotopes où l'espèce St. virei a été rencontrée. En B: nappes de pentes, sources de versants montagneux et biotope hypotelminorhéique, en A: milieu karstique (réseaux fossile et actif, avec des graviers formant un biotope interstitiel local), en C: vallée alluviale avec nappes parafluviales et sous-écoulement du cours d'eau. $\mathrm{P}=$ puits maçonnés, $\mathrm{p}=$ puits tubés. Les flèches rectilignes signalent les points où les Sténaselles sont capturés ou observés et ceux où ils sont supposés pouvoir vivre. Les flèches courbes matérialisent le trajet des eaux. Les différences écologiques entre les 5 sous-espèces de $S t$. virei sont rappelées. $\mathrm{R}=$ rivière. 


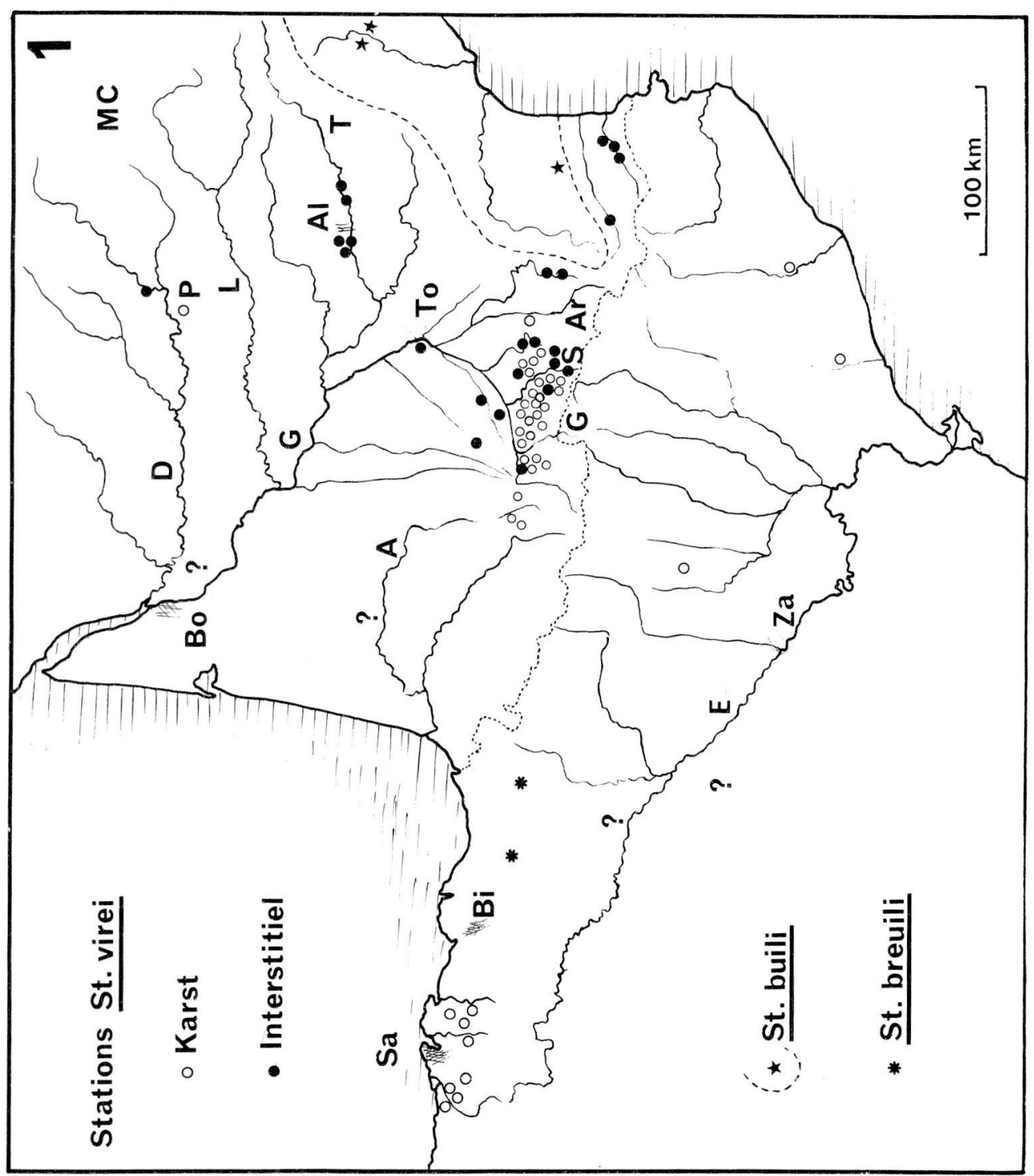




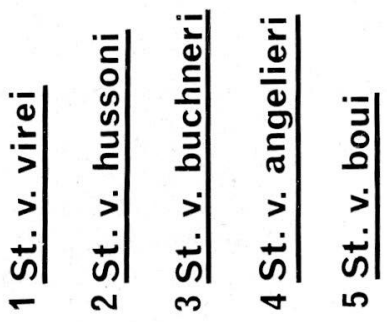

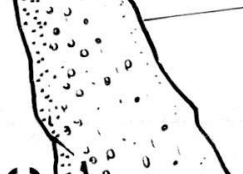

$0\left(\begin{array}{llll}0 & 0 & 0 \\ 0 & 0 & 0 & 0 \\ 0 & 0 & 1 & 0 \\ 0 & 0 & 0 & 0\end{array}\right.$
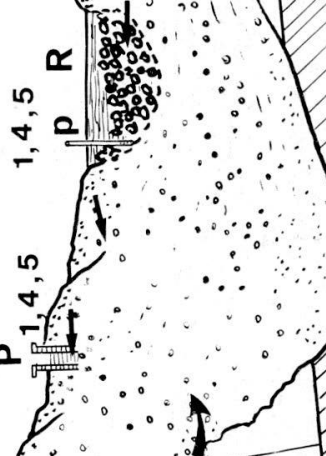

$\therefore$
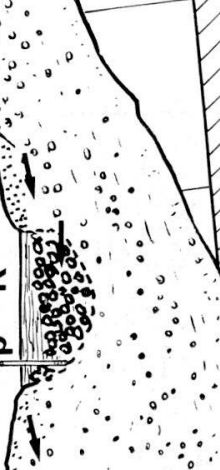

. 